\title{
WHITEHEAD SQUARES IN THOM COMPLEXES
}

\author{
by W. A. SUTHERLAND \\ (Received 23rd April 1980)
}

\section{Introduction}

One of the aims of this paper is to examine the following conjecture, attributed to Mahowald on p. 255 of (2), Part 2. Let $M$ be a closed connected smooth manifold of odd dimension $q(q \neq 1,3,7)$ and with tangent bundle $\tau$. Let the inclusion of a compactified fibre in the Thom complex of $\tau$ be written $\mu: S^{a} \rightarrow T_{\tau}$.

Conjecture A. The Whitehead square $[\mu, \mu]$ in $\pi_{2 q-1}(T \tau)$ is zero if and only if the Kervaire mod 2 semi-characteristic $\chi_{*}(M)$ is non-zero.

As we shall see, this is false in general, but it is true for a restricted class of manifolds including $\pi$-manifolds. (I have avoided calling it 'Mahowald's conjecture' since Mahowald knew it to be false. I am grateful to him for a conversation about this question. A misprint in (2) has been corrected here-the version printed in (2) is false even when $M$ is $S^{q}$.)

In (12), Marcum and Randall study $[\mu, \mu]$ more generally when $\mu: S^{q} \rightarrow T \xi$ is the analogous homotopy Thom class of any $q$-plane bundle $\xi$. In this paper we concentrate mainly on $\tau$ and one other special case, the normal bundle $\nu$ to an embedding of $M^{a}$ in Euclidean space $\mathbb{R}^{2 a}$. Also, we restrict $q$ to be odd, $q \neq 1,3,7$. (When $q$ is even, $[\mu, \mu]$ may be studied using Euler classes as in Proposition 2.1 of (12). If $q=1,3$ or 7 then $[\iota, \iota]=0$ for a generator $\iota$ of $\pi_{q}\left(S^{q}\right)$, so $[\mu, \mu]=\mu_{*}[\iota, \iota]=0$.) We use classical Hopf invariants, with an associated cohomological detection method developed by Browder and Dupont. There is some overlap of results with (12), but our method is slightly different, and it leads to a rather satisfactory answer for $\nu$. It is a pleasure to acknowledge the stimulus of (12) and of correspondence with Duane Randall. Since this paper was written, Larry Smith has kindly sent me a preprint in which he independently proves Proposition 2.5(a) by a rather different method.

We also relate the study of $[\mu, \mu]$ to work of James and Thomas in (11). One by-product (Proposition 2.3) is of separate interest as a footnote to (11), and gets a self-contained proof in the appendix. This was given at a meeting of the Edinburgh Mathematical Society, whose hospitality I acknowledge with gratitude.

\section{Statement of results}

All manifolds considered here are closed, connected, smooth and odd-dimensional. Cohomology is taken with mod 2 coefficients except that the 'degree' of a map means 
the usual degree in integral cohomology. We write $\mu$ for any homotopy Thom class and $U$ for any cohomology Thom class, wherever this is unlikely to cause confusion. Likewise, for any manifold $M^{a}$ we write $\tau$ for its tangent bundle and $\nu$ for the normal bundle to an embedding of $M^{a}$ in $\mathbb{R}^{2 a}$. We begin with results about $\nu$.

Theorem 2.1. (a) If $[\mu, \mu]=0$ in $T \nu$ then $q=2^{r}-1$ for some integer $r$.

(b) When $q=2^{r}-1$, there exists a $q$-dimensional manifold with $[\mu, \mu]=0$ in $T \nu$.

A suitable example for (b) is real projective space $\mathbb{R} P\left(2^{r}-1\right)$, or any manifold of dimension $2^{r}-1$ which admits a stable map to $\mathbb{R} P\left(2^{r}-1\right)$ of non-zero mod 2 degree. (In fact one can show that this accounts for all examples.) In particular there is an actual map of non-zero mod 2 degree from $\mathbb{R} P\left(n_{1}\right) \times \mathbb{R} P\left(n_{2}\right) \times \ldots \times \mathbb{R} P\left(n_{1}\right)$ to $\mathbb{R} P\left(2^{r}-1\right)$ provided $\sum n_{i}=2^{r}-1$ and the multinomal coefficient $\left(\left(2^{r}-1\right) ! ; n_{1} !, n_{2} !, \ldots, n_{t} !\right)$ is odd.

It is interesting to relate this discussion to (11). When $\xi$ is a $q$-plane bundle over a finite-dimensional complex $K$, let $j(\xi)$ be the 'fibre-homotopy James-Thomas number of $\xi$, i.e. the number of distinct fibre-homotopy equivalence classes of $(q-1)$-sphere bundles over $K$ which are stably fibre-homotopy equivalent to the sphere-bundle $S \xi$. When $K$ is a $q$-dimensional manifold ( $q$ odd) it is known that $j(\xi)$ is either 1 or 2 , and in particular for $\mathbb{R} P(q)$ we have $j(\nu)=1$ if and only if $q=2^{r}-1$ for some integer $r$ (see (11), (15).

Proposition 2.2. With the above notation, $[\mu, \mu]=0$ in $T \nu$ if and only if $j(\nu)=1$.

Proposition 2.3. If $j(\nu)=1$ then $q=2^{r}-1$ for some integer $r$.

Of course if we assume Proposition 2.2 then Theorem 2.1(a) and Proposition 2.3 imply each other.

Proposition 2.4. Suppose $j(\nu)=2$, and let $S \nu^{\prime}$ be a $(q-1)$-sphere bundle over $M^{a}$ which is stably, but not actually, fibre-homotopy equivalent to $S \nu$. Then $\left[\mu^{\prime}, \mu^{\prime}\right]=0$, where $\mu^{\prime}$ is the homotopy Thom class of $\nu^{\prime}$.

Our results about $\tau$ are less satisfying, but they do give an indication of the amount of truth in Conjecture A. For some of the discussion it is not essential to have a manifold-a $q$-dimensional complex with a unique $q$-cell will do. Let us call a $q$-plane bundle $\xi$ over such a complex stably mod 2 reducible if there exists a map from $S^{2 a+1}$ to the suspension $\Sigma T \xi$ inducing an isomorphism of (mod 2) cohomology in dimension $2 q+1$. For any manifold, $\nu$ is stably mod 2 reducible. A manifold $M$ will be called special (for want of a better name) if $\tau$ is stably mod 2 reducible. For example any $\pi$-manifold is special.

In the next two results, $w_{i}$ denotes the $i$ th mod 2 Stiefel-Whitney class of $\tau$.

Proposition 2.5. Suppose that either

(a) $M$ is a homotopy $\pi$-manifold, or

(b) $M$ is special, of dimension $q=2^{r}(2 m+1)-1$ with $r, m>0$, and $w_{i}=0$ for $1 \leqq i \leqq$ $2^{r}$.

Then Conjecture A holds for $M$. 
Notice that case (a) is included in case (b) unless $q+1$ is a power of 2 . An example in Section 7 shows that not all special manifolds obey Conjecture A.

Proposition 2.6. Suppose that $q$ is as in Proposition 2.5(b) and that $M^{q}$ is not special, but $w_{i}=0$ for $1 \leqq i \leqq 2^{r-1}$ and for $i=2^{r+1} m$. Then $[\mu, \mu] \neq 0$ in $T \tau$.

We need vanishing of Stiefel-Whitney classes to make our present proofs work. Examples in Section 7 indicate that such conditions may actually be needed to get any result like Proposition 2.6. Clearly Proposition 2.6 gives scope for Conjecture A to fail, and in Section 7 we give counterexamples. The conjecture could plausibly be weakened by adding the hypothesis $j(\tau)=2$ (to eliminate 'indeterminacy'); but Example 7.5 shows that the modified conjecture still fails in general.

Some of our techniques apply more generally. In the following results, $K$ is a $q$-dimensional complex, and for simplicity we assume that $K$ has a unique $q$-cell.

Proposition 2.7. Suppose that $S \xi$ and $S \xi^{\prime}$ are stably fibre-homotopy equivalent $(q-1)$-sphere bundles over $K$ and that $\xi$ is not stably mod 2 reducible. Let $\mu, \mu^{\prime}$ denote the homotopy Thom classes of $\xi$, $\xi^{\prime}$ respectively. Then $[\mu, \mu]=0$ if and only if $\left[\mu^{\prime}, \mu^{\prime}\right]=$ 0 .

Proposition 2.8. Suppose that $\xi$ is a stably mod 2 reducible q-plane bundle over $K$. Then either $[\mu, \mu]=0$ in $T \xi$ or $j(\xi)=2$.

In particular Proposition 2.8 shows that half of Proposition 2.2 (the implication $j(\nu)=1 \Rightarrow[\mu, \mu]=0$ ) holds more generally. The converse fails in general: for example if $\tau_{q}$ is the tangent bundle of $S^{q}(q$ odd, $q \neq 1,3,7)$ then $[\mu, \mu]=0$ in $T \tau_{q}$ and $S^{a}$ is special, but $j\left(\tau_{q}\right)=2$.

\section{Hopf invariants}

We recall some facts about Hopf invariants. Let $\xi$ be a $q$-plane bundle over a connected $q$-dimensional complex, $q$ odd. From (18) p. 548, part of the EHP exact sequence for $T \xi$ is:

$$
\ldots \rightarrow \pi_{2 q}(T \xi) \stackrel{\Xi}{\rightarrow} \pi_{2 q+1}(\Sigma T \xi) \stackrel{\leftrightarrow}{\rightarrow} \pi_{2 q+1}(\Sigma T \xi \wedge T \xi) \stackrel{P}{\rightarrow} \pi_{2 q-1}(T \xi) \rightarrow \ldots
$$

Here $\pi_{2 q+1}(\Sigma T \xi \wedge T \xi)$ is infinite cyclic or of order 2 according as $\xi$ is orientable or not. Let $\mathrm{H}_{2}$ denote the Hopf invariant in the latter case and its mod 2 reduction in the former case. Comparison with the EHP sequence for $S^{a}$ shows that $[\mu, \mu]$ generates the image of $P$ in (3.1) and is zero if and only if there exists a map $f: S^{2 q+1} \rightarrow \Sigma T \xi$ with $\mathrm{H}_{2}(f) \neq 0$. The method for calculating $\mathrm{H}_{2}(f)$ is due to Steenrod in the case of spheres, but in our case it is convenient to quote (4). Let $U$ denote the Thom class in $H^{a}(T \xi)$. According to Corollary 5.15 of $(\mathbf{4}), \mathrm{H}_{2}(f)$ is detected by the functional Steenrod square $S q_{f}^{q+1}(\Sigma U)$ provided the latter has zero indeterminacy, i.e. provided $f$ induces the zero homomorphism $f^{*}$ on $H^{2 a+1}$. However, even when $f^{*} \neq 0$ we may still detect $\boldsymbol{H}_{2}(f)$ by a functional square following Browder (5). Let $K_{\mathrm{q}}$ denote an Eilenberg-MacLane space 
of type $(\mathbb{Z} / 2, q)$ with fundamental cohomology class $\iota$, and consider $U$ as a map from $T \xi$ to $K_{q}$. By Section 1 of $(5)$ and Corollary 5.15 of $(4), H_{2}(f)$ is detected by $S q_{\Sigma}^{q+1}\left(\Sigma_{\imath}\right)$, which has zero indeterminacy.

\section{Normal bundles}

As before let $\nu$ be the normal bundle to an embedding of a manifold $M^{q}$ in $\mathbb{R}^{2 q}, q$ odd. The following easy lemma simplifies the study of $[\mu, \mu]$ in $T \nu$.

Lemma 4.1. The degree in dimension $2 q+1$ of any map $f: S^{2 q+1} \rightarrow \Sigma T \nu$ may be changed arbitrarily without changing the Hopf invariant $H(f)$.

Proof. Let $a: S^{2 q} \rightarrow T \nu$ be obtained from an embedding of $M$ in $\mathbb{R}^{2 a}$ via the Pontrjagin-Thom construction. It is well known that $a$ has degree 1 in dimension $2 q$, and $H(\Sigma a)=0$ by (3.1). Since $H$ is a homomorphism, $H(f+\Sigma a)=H(f)$ and the lemma follows.

We may now prove Theorem 2.1(a). If $[\mu, \mu]=0$ in $T \nu$ then by (3.1) there exists a map $f: S^{2 a+1} \rightarrow \Sigma T \nu$ with $H_{2}(f) \neq 0$. By Lemma 4.1 we may assume that $f$ has even degree, so $S q_{f}^{q+1}(\Sigma U)$ has zero indeterminacy and by Section $3, S q_{f}^{q+1}(\Sigma U) \neq 0$ since $H_{2}(f) \neq 0$. From an $S$-dual of $f$ we obtain a map $g: \Sigma^{n} M \rightarrow S^{n}$ for $n$ large. Let $s$ generate $H^{n}\left(S^{n}\right)$ and let $\chi$ denote the canonical anti-automorphism of the mod 2 Steenrod algebra. Then by $S$-duality, $\left(\chi S q^{q+1}\right)_{\mathrm{g}}(s) \neq 0$ and exactly as in the proof of Lemma 2.3 in (6) it follows that $q=2^{r}-1$ for some integer $r$.

To prove Theorem $2.1(\mathrm{~b})$, recall from (3) that if $M=\mathbb{R} P(q)$ then stably $T \nu$ is the stunted projective space

$$
P_{k-q-1}^{k-1}=\mathbb{R} P(k-1) / \mathbb{R} P(k-q-2) .
$$

Here $k$ is the order of the reduced Grothendieck group $\widetilde{K O}(\mathbb{R} P(q))$. Let $f$ be the map which, when suitably suspended, attaches the top cell in $P_{k-q-1}^{k}$. We may compute $S q_{f}^{q+1}(\Sigma U)$ by applying $S q^{q+1}$ to the generator of $H^{k-q-1}\left(P_{k-q-1}^{k}\right)$. A familiar calculation shows that this is non-zero if (and only if) $q+1=2^{r}$ for some integer $r>3$, and Theorem 2.1(b) follows by Section 3. (Theorem 2.1(b) still holds when $r=1,2$ or 3 since then $[\iota, \iota]=0$.)

To verify the remark after Theorem 2.1 , note that if $\theta: M \rightarrow \mathbb{R} P(q)$ is an $S$-map inducing an isomorphism of cohomology in dimension $q$, then its $S$-dual yields a map $\psi: \Sigma T \nu(\mathbb{R} P(q)) \rightarrow \Sigma T \nu(M)$ preserving $\Sigma U$. Hence if $f: S^{2 q+1} \rightarrow \Sigma T \nu(\mathbb{R} P(q))$ has even degree and $H_{2}(f) \neq 0$, then $\psi \circ f$ has the same properties so $[\mu, \mu]=0$ in $T \nu(M)$ by (3.1).

\section{Connection with James-Thomas number}

We begin by recalling some facts about fibre-homotopy automorphisms which are key ingredients in our proof of Proposition 2.2. The first is a result of Dupont in (10) which may be formulated as follows. Let $K$ be a $q$-dimensional complex with a unique $q$-cell, $q$ odd, and let $\xi$ be a $q$-plane bundle over $K$. Let $\alpha$ be a stable fibre-homotopy automorphism of $S \xi$. We may think of $\alpha$ alternatively as a fibring over $S^{1} \times K$, so $w_{q+1}(\alpha)$ is a well-defined element of $H^{q+1}\left(S^{1} \times K\right)$. Thus $w_{q+1}(\alpha)$ may be evaluated on 
the fundamental homology class of $S^{1} \times K$ to yield a mod 2 number $\chi(\alpha)$. Suppose that $X$ is a complex with $H^{2 q+n}(X) \approx \mathbb{Z} / 2$ for some large $n$ and $S q^{q+1} H^{q+n-1}(X)=0$. Let $f: X \rightarrow \Sigma^{n} T \xi$ satisfy

(a) $f^{*}\left(\Sigma^{n} U\right)=0$

(b) $f^{*}: H^{n+2 a}\left(\Sigma^{n} T \xi\right) \rightarrow H^{n+2 a}(X)$ is an isomorphism.

Notice that $T \alpha$ of also satisfies (a) and (b), where $T \alpha$ is the map of $\Sigma^{n} T \xi$ induced by $\alpha$. As in Section 3 let us represent the cohomology Thom class of $\xi$ by a map $U$ from $T \xi$ to an Eilenberg-MacLane space with fundamental class $\iota$. Then Proposition 4.4 of (10) yields:

$$
S q_{\Sigma^{n} U \circ T \alpha \circ f}^{q+1}\left(\Sigma^{n} \imath\right)-S q_{\Sigma^{n} U \circ f}^{q+1}\left(\Sigma^{n} \iota\right)=\chi(\alpha) \text {. }
$$

In (10), $K=M$ is a manifold, $X$ is a space in a Wu cospectrum, and in the applications $S \xi$ is stably fibre-homotopy equivalent to $S \tau$. But (5.1) applies equally well to other cases: for example if $\xi$ is stably mod 2 reducible we may take $X=S^{2 q+n}$ and there is a map $f: S^{2 a+n} \rightarrow \Sigma^{n} T \xi$ satisfying (a) and (b).

We need also the following theorem.

Theorem 5.2. $((\mathbf{1 0}),(\mathbf{1 1}))$. Let $\xi$ be a $q$-plane bundle over a $q$-dimensional complex with a unique $q$-cell, $q$ odd. Then $j(\xi)=1$ and only if there exists a stable fibrehomotopy automorphism $\alpha$ of $S \xi$ with $w_{\mathrm{q}+1}(\alpha) \neq 0$.

This follows from (10), Corollary 2.3, Definition 2.4 and Proposition 2.7. Alternatively, it is a reformulation of Theorem 1.6 of (11).

The final ingredient is a result of Wall relating degree 1 maps from $S^{2 a+n}$ to $\Sigma^{n} T \nu$ with stable fibre-homotopy automorphisms of $S \nu$ (over the identity map of $M$ ). Any such automorphism $\alpha$ gives rise to a homotopy equivalence $T \alpha: \Sigma^{n} T \nu \rightarrow \Sigma^{n} T \nu$ for $n \geqq 1$ (unique up to homotopy for $n \geqq 2$ ). By Theorem 3.5 of (17) any choice of degree 1 map $f: S^{2 q+n} \rightarrow \Sigma^{n} T \nu$ determines a one-one correspondence $\alpha \rightarrow T \alpha$ of between homotopy classes of stable fibre-homotopy automorphisms of $S \nu$ and homotopy classes of stable maps of degree 1 from $S^{2 q+n}$ to $\Sigma^{\mathfrak{n}} T \nu$.

Proof of Proposition 2.2. To establish the 'if' part we prove the more general Proposition 2.8. Suppose $\xi$ is as in Proposition 2.8 and suppose $j(\xi)=1$. Let $g: S^{2 a+1} \rightarrow$ $\Sigma T \xi$ induce an isomorphism of $H^{2 a+1}$. By Theorem 5.2 there exists a stable fibrehomotopy automorphism $\alpha$ of $S \xi$ with $w_{q+1}(\alpha) \neq 0$. Now apply (5.1), taking $X=S^{2 a+n}$ and $f=\Sigma^{n-1} g$. Since $\chi(\alpha) \neq 0$, one of the terms on the left-hand side of $(5.1)$ must be non-zero, so either $g$ or a desuspension of $T \alpha$ of provides a map, $h$ say, from $S^{2 q+1}$ to $\Sigma T \xi$ with $H_{2}(h) \neq 0$. Hence $[\mu, \mu]=0$ in $T \xi$ by (3.1). This proves Proposition 2.8 and $a$ fortiori one half of Proposition 2.2.

Conversely suppose $[\mu, \mu]=0$ in $T \nu$. By (3.1) there exists a map $f: S^{2 a+1} \rightarrow \Sigma T \nu$ with $H_{2}(f) \neq 0$, and by Lemma 4.1 we may assume $f$ has degree 1 . Let $a$ be as in the proof of Lemma 4.1, so that $\Sigma a: S^{2 a+1} \rightarrow \Sigma T \nu$ has degree 1 and $H(\Sigma a)=0$. By Wall's result recalled above, there exists a stable fibre-homotopy automorphism $\alpha$ of $S \nu$ such that $T \alpha \circ \Sigma a=f$. Then $\chi(\alpha) \neq 0$ by (5.1), so $j(\nu)=1$ by Theorem 5.2.

Proposition 2.4 can be proved in the above style (as can Proposition 2.7, whose proof is omitted), but I prefer the following proof, pointed out to me by Duane Randall. I am very grateful for his permission to use it here. 
Proof of Proposition 2.4. (Randall). Suppose that $j(\nu)=2$. Take a cellular decomposition $M=M_{O} \cup e^{q}$ with a unique $q$-cell. Choose classifying maps $f, f^{\prime}: M \rightarrow B O(q)$ for $\nu, \nu^{\prime}$ respectively, with $f=f^{\prime}$ on $M_{O}$. since $i(\nu)=2$, the difference class $d\left(\nu, \nu^{\prime}\right)$ in $\pi_{q}(B O(q)) \approx \pi_{q-1}(O(q))$ is non-zero. On the other hand, $S \nu$ and $S \nu^{\prime}$ are stably fibre-homotopy equivalent. Putting these facts together we get $J d\left(\nu, \nu^{\prime}\right)=[\iota, \iota]$ where $J: \pi_{q-1}(O(q)) \rightarrow \pi_{2 q-1}\left(S^{q}\right)$ is the usual $J$-homomorphism. Let $\nu_{O}=\nu \mid M_{O}$ and let $\mu_{O}, \mu, \mu^{\prime}$ be the homotopy Thom classes of $\nu_{O}, \nu, \nu^{\prime}$ respectively. Since the attaching map for the top cell in $T \nu$ is zero in $\pi_{2 q-1}\left(T \nu_{O}\right)$, it follows from Theorem $D$ of (14) that the attaching map for the top cell in $T \nu^{\prime}$ is $\mu_{O *} J d\left(\nu, \nu^{\prime}\right)=\mu_{O *}[\iota, \iota]=\left[\mu_{0}, \mu_{0}\right]$. Hence $\left[\boldsymbol{\mu}^{\prime}, \mu^{\prime}\right]=0$.

Remark. Duane Randall has pointed out that some other results in this section may alternatively be proved by the above technique and Browder-Levine embedding theory.

\section{Tangent bundles}

First we prove Proposition 2.6. Suppose $M^{a}$ is not special, but $w_{i}=0$ for $1 \leqq i \leqq 2^{r-1}$ and for $i=2^{r+1} m$, where $q=2^{r}(2 m+1)-1, r, m>0$. Let $f$ be any map from $S^{2 q+1}$ to $\Sigma T \tau$. We shall prove $H_{2}(f)=0$; the result then follows by (3.1).

Since $M$ is not special, $f$ has even degree, so $S q_{f}^{q+1}(\Sigma U)$ has zero indeterminacy and detects $\mathrm{H}_{2}(f)$. Now we use the Adem decomposition

$$
S q^{q+1}=S q^{2 r} S q^{2 r+1 / m}+\sum b_{j} S q^{q+1-j} S q^{j}
$$

Here $b_{j}$ is a mod 2 binomial coefficient and $j$ ranges from 1 to $2^{r-1}$. Since for $1 \leqq j \leqq 2^{r-1}$ and for $j=2^{r+1} m$ we have $S q_{f}^{j}(\Sigma U)$ defined and zero (with zero indeterminacy) it follows that $S q_{f}^{q+1}(\Sigma U)=0$ as required.

Remark. Duane Randall has pointed out that Proposition 2.6 may equivalently be proved by applying (directly to $T_{\tau}$ ) a secondary operation based on (6.1).

Next we prove Conjecture A for a homotopy $\pi$-manifold i.e. assuming $S \tau$ is stably fibre-homotopy trivial. In this case $S \tau$ is fibre-homotopy trivial if and only if $\chi_{*}(M)=0$ (see for example (9), and recall that $q$ is odd, $q \neq 1,3,7)$. Thus if $\chi_{*}(M)=0$ then $S \tau$ is fibre-homotopy trivial and $[\mu, \mu] \neq 0$ in $T \tau$ since otherwise $[\iota, \iota]=0$ in $\pi_{2 q-1}\left(S^{q}\right)$. On the other hand if $\chi_{*}(M) \neq 0$ then by (9), Proposition 3.4 and Definition 3.1, there exists a map $f: S^{2 q+1} \rightarrow \Sigma T \tau$ with $H_{2}(f) \neq 0$ so $[\mu, \mu]=0$ in $T \tau$ by (3.1).

Before proving Conjecture A under assumption 2.5(b), we discuss special manifolds briefly. For $M$ special, there exists a map from $S^{2 a+1}$ to $\Sigma T_{\tau}$ of odd degree. Equivalently by $S$-duality there exists a map from $T\left(2 \nu_{s t}\right)$ to $S^{2 n}$ of odd degree, where $\nu_{s t}$ is an $n$-plane normal bundle of $M$ with $n$ large. Then by ((3), proof of Proposition 2.8) and $\left((\mathbf{1})\right.$, Theorem (1.1)), it follows that $2 v_{s t}$ has odd order in the fibre-homotopy sense. (The converse is also true-see (8).) In particular it is easily checked that $w_{i}=\bar{w}_{i}$ for all $i$, where $\bar{w}_{i}$ is the dual Stiefel-Whitney class.

Lemma 6.2. If $M^{a}$ is special, $q=2^{r}(2 m+1)-1$ with $r, m>0$ and $w_{i}=0$ for $1 \leqq i \leqq$ $2^{r-2}$ then $w_{i}=0$ for $i=2^{r+1} m$. 
Proof. This may be proved for the dual Stiefel-Whitney classes in a straightforward fashion following the method in (13).

To see that special manifolds are rather scarce, note that if any stable mod 2 cohomology operation arriving in $H^{2 a}(T \tau)$ is non-zero then $M$ is not special.

Proof of Proposition 2.5(b). Suppose that $M$ satisfies $2.5(\mathrm{~b})$ and suppose that $[\mu, \mu]=0$ in $T \tau$. As in the proof of Proposition 2.2 of (12), let $g: S^{a} \cup_{[\omega, L]} e^{2 a} \rightarrow T \tau$ extend $\mu$. By hypothesis, a secondary operation $\Phi$ associated with (6.1) is defined on $U_{\tau}$. Also, $\Phi$ detects $[\iota, \iota]$ by (7), so $\Phi\left(U_{\tau}\right) \neq 0$. Now, for example by (16), Remark (b) on p. 111 , we get $\chi_{*}(M) \neq 0$.

For the converse we use the Browder-Dupont method described in (16). Let $X^{\prime}$ be as in Section 8 of (16): recall then $H^{2 a+n}\left(X^{\prime}\right) \approx \mathbb{Z} / 2$ and

$$
S q^{i} H^{2 q+n-i}\left(X^{\prime}\right)=0 \text { for } 1 \leqq i \leqq 2^{r} \text { and for } i=q+1
$$

Suppose $\chi_{*}(M) \neq 0$. Then by $(16$, Section 8$)$ and Theorem 2.7 , there is a map $g^{\prime}: X^{\prime} \rightarrow \Sigma^{n} T_{\tau}$ (an allowable $X^{\prime}$-orientation) inducing an isomorphism of $H^{2 q+n}$ and with $S q_{\Sigma^{n} U^{q} g^{\prime}}^{q+1}\left(\Sigma^{n} \iota\right) \neq 0$. Since $M$ is special, there exists a map $f: S^{2 a+1} \rightarrow \Sigma T \tau$ of odd degree. Suppose, for a contradiction, that $H_{2}(f)=0$. We may compose $\Sigma^{n-1} f$ with a map of odd degree from $X^{\prime}$ to $S^{2 q+n}$ to get $h: X^{\prime} \rightarrow \Sigma^{n} T_{\tau}$ such that $S q_{\Sigma^{n} U^{+a h}}^{q+1}\left(\Sigma^{n} l\right)=0$. Then $g=g^{\prime}+h$ satisfies $S q_{\Sigma^{n} U \circ g}^{q+1}\left(\Sigma^{n} \iota\right) \neq 0$ and $g$ induces the zero homomorphism on $H^{2 a+n}$, so $S q_{\mathrm{g}}^{a+1}\left(\Sigma^{n} U\right) \neq 0$ with zero indeterminacy. But each term $S q^{a+1-i}$ on the right-hand side of (6.1) lies in the right ideal of the mod 2 Steenrod algebra generated by the $S q^{i}$ with $1 \leqq i \leqq 2^{r-1}$ (cf. (16) p. 110). By hypothesis and Lemma $6.2, S q_{g}^{j}\left(\Sigma^{n} U\right)$ is defined for $1 \leqq j \leqq 2^{r-1}$ and for $j=2^{r+1} \mathrm{~m}$. Using (6.1) and (6.3) we now get the contradiction $S q_{\mathrm{g}}^{q+1}\left(\Sigma^{n} U\right)=0$. Hence $H_{2}(f) \neq 0$, so $[\mu, \mu]=0$ in $T \tau$ by (3.1).

\section{Examples}

In this section we present some examples relevant to the previous results. Proposition 2.6 suggests how to get counterexamples to Conjecture A. However, we begin with a simple counterexample which does not satisfy all the hypotheses of Proposition 2.6.

Example 7.1. Let $M=\mathbb{R} P(11)$. Then $\chi_{*}(M)=0$. We shall prove $[\mu, \mu]=0$ in $T \tau$ by exhibiting a map $f: S^{23} \rightarrow \Sigma T \tau$ with $H_{2}(f) \neq 0$. In the notation of Section $4, \Sigma T \tau=P_{12}^{23}$. Let $f$ be the attaching map for the top cell in $P_{12}^{24}$. Since $f$ has even degree and $S q^{12} \neq 0$ in $P_{12}^{24}$ it follows that $H_{2}(f) \neq 0$. In this argument, 11 may be replaced by $4 k+3$ for any positive integer $k$.

In Example 7.1, $M$ is not special and $w_{i} \neq 0$ for $i=2^{r+1} m$. In fact when $M$ is not special and we do not insist on $w_{i}$ vanishing as in Proposition 2.6 , literally anything can happen concerning the values of $\chi_{*}(M)$ and of $[\mu, \mu]$ in $T \tau$. To illustrate this we give three further examples, omitting details.

Example 7.2. If $M=\mathbb{C} P(4 k) \times S^{3}$ then $[\mu, \mu] \neq 0$ in $T \tau$ and $\chi_{*}(M) \neq 0$.

Example 7.3. If $M=\mathbb{R} P(8 k+1) \times S^{2}$ then $[\mu, \mu] \neq 0$ in $T \tau$ and $\chi_{*}(M)=0$. 
Example 7.4. If $M$ is the total space of the $S^{3}$-bundle $S \eta$ over $\mathbb{Q} P(2)$ in the notation of $(3)$ then $[\mu, \mu]=0$ in $T_{\tau}$ and $\chi_{*}(M) \neq 0$.

In Example 7.2, $j(\tau)=1$. With the next example, this shows that there is no analogue of Proposition 2.2 for tangent bundles in general.

Example 7.5. If $M=\mathbb{R} P(2) \times S^{4 k-1}(k>2)$ then $[\mu, \mu]=0$ in $T \tau, \chi_{*}(M) \neq 0$ and $j(\tau)=2$.

Example 7.5 shows that even if we add the hypothesis $j(\tau)=2$ to Conjecture A, the modified conjecture is still false in general. The next example illustrates Proposition 2.6.

Example 7.6. If $M=\mathbb{Q} P(2 k) \times S^{9}$ then $w_{i}=0$ for $i=1,2,8 k+8, \chi_{*}(M) \neq 0$ and $[\mu, \mu] \neq 0$ in $T \tau$.

The final example shows that not all special manifolds obey Conjecture A.

Example 7.7. If $M=K \times S^{13}$ where $K$ is the Klein bottle, then $M$ is special (I am grateful to $\operatorname{Reg}$ Wood for pointing this out), $\chi_{*}(M)=0$ and $j(\tau)=j(\nu)=1$, hence $[\mu, \mu]=0$ in $T \tau$ by Proposition 2.2 .

I do not know wheather all special manifolds with $j(\tau)=2$ obey Conjecture A. It is easy to show (using Proposition 2.3) that $j(\tau)=2$ for any special manifold whose dimension is not of the form $2^{r}-1$.

\section{Appendix}

Here a self-contained proof of Proposition 2.3.

Let $\xi$ be a $q$-plane bundle over a manifold $M^{q}$ ( $q$ odd). As before we write $\xi_{s t}$ for the stable class of $\xi$. By Theorem 5.2, $j(\xi)=1$ if and only if there exists a stable sphere fibring $\alpha$ over $S^{1} \times M$ with $w_{\mathrm{q}+1}(\alpha) \neq 0$ and of 'type' $\left(0, \xi_{s t}\right)$, i.e. the restriction of $\alpha$ to the wedge $S^{1} \vee M$ is $0 \vee \xi_{s t}$. Suppose there exists such an $\alpha$. Then $S q^{q+1} \neq 0$ in $T \alpha$. By $S$-duality, the stable sphere fibring $\beta=\nu_{s t}-\alpha$ is of type $\left(0, \nu_{s t}-\xi_{s t}\right)$, and $\chi S q^{q+1} \neq 0$ in $T \beta$, i.e. the Wu class $v_{q+1}(\beta) \neq 0$. In particular if $\xi_{s t}=\nu_{s t}$ then $\beta$ is of type $(0,0)$, hence is induced from a bundle $\gamma$ over $\Sigma M$ with $v_{q+1}(\gamma) \neq 0$. It is well known that $v_{q+1}$ is decomposable unless $q+1$ is a power of 2 , and the result follows since cup-products vanish on a suspension. This last argument has been used by Brown in (6) p. 381 and by Jones and Rees in (2), Part I, p. 144.

\section{REFERENCES}

(1) J. F. AdAMs, On the groups $J(X)-I$, Topology 2 (1963), 181-95.

(2) A.M.S. Colloquia Proceedings, Vol. 32 (Providence, R.I., 1978).

(3) M. F. Aтryah, Thom complexes, Proc. London Math. Soc. (3) 11 (1961), 291-310.

(4) J. M. Boardman and B. Steer, On Hopf invariants, Comment. Math. Helv. 42 (1967), 180-221.

(5) W. Browder, The Kervaire invariant of framed manifolds and its generalizations, Ann. of Math. 90 (1969), 157-86. 
(6) E. H. Brown JNR., Generalizations of the Kervaire invariant, Ann. of Math. 95 (1972), 368-83.

(7) E. H. Brown JNR and F. P. PEterson, Whitehead products and cohomology operations, Quart. J. Math. (Oxford) (2) 15 (1964), 116-20.

(8) I. Dibag, Degree theory for spherical fibrations, to appear.

(9) J. L. Dupont, On homotopy invariance of the tangent bundle I, Math. Scand. 26 (1970), 5-13.

(10) J. L. Dupont, On homotopy invariance of the tangent bundle II, Math. Scand. 26 (1970), 200-220.

(11) I. M. JAmEs and E. Thomas, An approach to the enumeration problem for non-stable vector bundles, J. Math. Mech. 14 (1965), 485-506.

(12) H. J. Marcum and D. Randall, The homotopy Thom class of a spherical fibration, Proc. Amer. Math. Soc. 80 (1980), 353-358.

(13) W. S. Massey and F. P. Peterson, On the dual Stiefel-Whitney classes of a manifold, Bol. Soc. Mat. Mexicana (2) 8 (1963), 1-13.

(14) R. J. Mrgram and Elmer Rees, On the normal bundle to an embedding, Topology 10 (1971), 299-308.

(15) W. A. SUTHERLaND, The fibre homotopy enumeration of non-stable sphere bundles and fibrings over real projective spaces, J. London Math. Soc. (2) 1 (1969), 693-704.

(16) W. A. Sutherland, The Browder-Dupont invariant, Proc. London Math. Soc. (3) 33 (1976), 94-112.

(17) C. T. C. WALl, Poincaré complexes: I, Ann. of Math. 86 (1967), 213-245.

(18) G. W. WHITEHEAD, Elements of homotopy theory (Springer-Verlag, 1978).

\section{New College}

OXFORD

OX1 3BN 